\title{
Upregulated PPARG2 facilitates interaction with demethylated AKAP12 gene promoter and suppresses proliferation in prostate cancer
}

\author{
Feng Li $\mathbb{B}^{1,2,3}$, Tingting Lư ${ }^{1,3}$, Dongmei Liu ${ }^{1,3}$, Chong Zhang ${ }^{3}$, Yonghui Zhang ${ }^{3}$ and Fulu Dong ${ }^{1,3}$
}

\begin{abstract}
Prostate cancer (PCA) is one of the most common male genitourinary tumors. However, the molecular mechanisms involved in the occurrence and progression of PCA have not been fully clarified. The present study aimed to investigate the biological function and molecular mechanism of the nuclear receptor peroxisome proliferatoractivated receptor gamma 2 (PPARG2) in PCA. Our results revealed that PPARG2 was downregulated in PCA, and overexpression of PPARG2 inhibited cell migration, colony formation, invasion and induced cell cycle arrest of PCA cells in vitro. In addition, PPARG2 overexpression modulated the activation of the Akt signaling pathway, as well as inhibited tumor growth in vivo. Moreover, mechanistic analysis revealed that PPARG2 overexpression induced increased expression level of miR-200b-3p, which targeted $3^{\prime}$ UTR of the downstream targets DNMT3A/3B, and facilitated interaction with demethylated AKAP12 gene promoter and suppressed cell proliferation in PCA. Our findings provided the first evidence for a novel PPARG2-AKAP12 axis mediated epigenetic regulatory network. The study identified a molecular mechanism involving an epigenetic modification that could be possibly targeted as an antitumoral strategy against prostate cancer.
\end{abstract}

\section{Introduction}

Prostate cancer (PCA) is one of the most common male genitourinary tumors with its high fatality rate in the western world ${ }^{1}$. Reports revealed that the United States is expected to spend more than US\$8 billion annually on the screening and treatment of $\mathrm{PCA}^{2}$. Incidence of PCA in China is lower than in the United States; however, in the past two decades, numbers of PCA patients have increased significantly due to environmental pollution, westernized change in diet, and aging of the population ${ }^{3}$. The prostate-specific antigen (PSA) is currently

\footnotetext{
Correspondence: Fulu Dong (fldste@163.com)

'Department of Pathology, Key Laboratory of Microenvironment and

Translational Cancer Research, Medical School of Nantong University, Nantong 226001 Jiangsu, China

2Department of Laboratory Medicine, Affiliated Hospital of Nantong University, Nantong 226001 Jiangsu, China

Full list of author information is available at the end of the article

These authors contributed equally: Feng Li, Tingting Lu
}

Edited by F. Pentimall recognized as a useful tool for early screening of $\mathrm{PCA}^{4}$. However, screening based on serum PSA is still largely debated $^{5}$, and up to $22 \%$ of newly diagnosed patients with PCA are advanced or metastatic ones ${ }^{6}$. Once diagnosed, it is usually treated by active surveillance, prostatectomy, radiation therapy, hormone therapy, or chemotherapy ${ }^{7}$. So far, the complex molecular mechanisms involved in the occurrence and progression of PCA have not been fully clarified. We believe it is necessary to explore the pathological mechanism and look for new molecular therapeutic targets of PCA.

The nuclear receptor peroxisome proliferator-activated receptor- $\gamma$ (PPARG) is a ligand-dependent transcription factor (TF) that plays a vital role in regulating the differentiation of adipocytes and the transcription of multiple genes $^{8-11}$. The human PPARG gene was found to be located on the short arm of chromosome 3 (3p25) in 1995. PPARG exists in two protein isoforms, PPARG1 and PPARG $2{ }^{11}$. Compared to PPARG1, PPARG2 contains 30

\section{(c) The Author(s) 2021}

(c) (i) Open Access This article is licensed under a Creative Commons Attribution 4.0 International License, which permits use, sharing, adaptation, distribution and reproduction c. in any medium or format, as long as you give appropriate credit to the original author(s) and the source, provide a link to the Creative Commons license, and indicate if changes were made. The images or other third party material in this article are included in the article's Creative Commons license, unless indicated otherwise in a credit line to the material. If material is not included in the article's Creative Commons license and your intended use is not permitted by statutory regulation or exceeds the permitted use, you will need to obtain permission directly from the copyright holder. To view a copy of this license, visit http://creativecommons.org/licenses/by/4.0/. 
additional amino acids at the $\mathrm{N}$ terminus and the ligandindependent activation activity is 5-10 times than that of PPARG $1^{12}$. It has been reported that PPARG plays a role in a variety of chronic diseases including tumors ${ }^{3,13}$, diabetes $^{14}$, inflammation ${ }^{15}$, atherosclerosis ${ }^{16}$, and so on. As far as PPARG2 is concerned, its role in PCA has not been clarified.

DNA methylation is a common type of epigenetic modification. The existence of $\mathrm{CpG}$ islands in human genome is always closely related to the methylation status and it is also related to a majority of the coding genes in the human genome ${ }^{17}$. DNA methylation plays an important role in the of gene expression, cell proliferation, differentiation, and development, and is also closely related to human development and tumors ${ }^{18-20}$. When one gene promoter region is methylated, its transcription is often inactivated, whereas demethylation is usually manifested as a transcriptional activation.

In the present study, we showed that the downregulation of PPARG2 expression in PCA acted as a tumor suppressor in suppressing malignancy of PCA cells in vitro and in vivo. Moreover, mechanistic analysis revealed that upregulated PPARG2 facilitated interaction with demethylated A-Kinase anchoring protein 12 (AKAP12) gene promoter and suppressed cell proliferation in PCA. Our present results provide the first evidence for a novel PPARG2-AKAP12 axis-mediated epigenetic regulatory network. The study identified a molecular mechanism involving an epigenetic modification that could be possibly targeted as an antitumoral strategy against PCA.

\section{Materials and methods \\ Cell lines and culture}

The human PCA cell lines (LNCap, PC3, and DU145) were purchased from the Chinese Academy of Sciences (Shanghai, China) and were cultured with Roswell Park Memorial Institute 1640 (10-040-CV, Corning, USA) containing $10 \%$ fetal bovine serum (FBS, Gibco, Billings, $\mathrm{MT}$ ). The non-malignant immortalized human prostate epithelial cell line named NHPrE1 has been described previously ${ }^{21,22}$. The cell line was routinely passaged in 50/ 50 Dulbecco's modified Eagle's medium (DMEM)/F12 (Gibco) culture medium containing 5\% FBS, 1\% insulintransferrin-selenium-X (Gibco), $0.4 \%$ bovine pituitary extract (Hammond Cell Tech, Windsor, CA), and $10 \mathrm{ng} /$ $\mathrm{ml}$ epidermal growth factor (Sigma, Woodstock, VA) with 1\% AB/AM (Gibco). The miR-200b-3p mimic and inhibitor were purchased from GenePharma Co., Ltd (Shanghai, China). HEK 293T cell line was cultured with DMEM containing 10\% FBS. Cells were cultured on different sizes of cell culture dishes in a humidified atmosphere containing $5 \% \mathrm{CO}_{2}$ at $37^{\circ} \mathrm{C}$. All cells were authenticated by short tandem repeat profiling.

\section{Tissue samples}

Eight human PCA samples and eight benign prostatic hyperplasia tissues were obtained from the Urology Department of Affiliated Hospital of Nantong University, between 2017 and 2018. The samples were frozen quickly in liquid nitrogen and were stored at $-80^{\circ} \mathrm{C}$. The study was in accordance with the International Ethical Guidelines for Biomedical Research Involving Human Subjects. The protocol was approved by the Ethics Committee of the Affiliated Hospital of Nantong University. All subjects obtained informed consent to participate in this study.

\section{Construction of lentivirus vectors}

The PPARG2 overexpression lentivirus vector-GV358 containing the human PPARG2 wild-type full-length sequence (PPARG2) for gain-of-function and the lentivirus empty vector $(\mathrm{EV})$ as a control were constructed by GeneChem Co., Ltd (Shanghai, China). In brief, the successful construction of the plasmid was first verified by restriction enzyme digestion, PCR identification, and sequencing. Then, the constructed plasmid and lentivirus packaging plasmids phelper 1.0 and phelper 2.0 were cotransfected into the cultured HEK 293T cells. Lentiviral particles were obtained by collecting supernatant using the kit for ultracentrifugation concentration and purification of lentiviral particles, and combined with fluorescent titer assay and enzyme-linked immunosorbent assay. Virus titer was determined as $1 \times 10^{8}$ transducing $\mathrm{U} \mathrm{ml}^{-1}$.

\section{Gene expression profiling and miRNA-seq analysis}

The gene expression profiles of PC3-PPARG2 cDNA (PPARG2) and PC3-EV were compared using Agilent SurePrint G3 Human Gene Expression $8 \times 60 \mathrm{~K}$ Microarray (Agilent Technologies, Santa Clara, CA) (Gene Expression Omnibus database accession number GSE108309). High-throughput miRNA sequencing (miRNA-seq) between PPARG2 and EV group was conducted using the single-ended $50 \mathrm{bp}$ sequencing mode of the Illumina Hiseq3000 sequencing platform (Genergy Bio-technology, Shanghai, China). The Sequence Read Archive (SRA) accession number was PRJNA719139. Differential expression genes (DEGs) between PPARG2 and EV were screened, based on a $t$-test of linear models for microarray analysis package in $\mathrm{R}$ (Version 3.3, http:// www.bioconductor.org) ${ }^{23}$. DEGs fold change of gene expression was calculated with a threshold of fold change $>1$ and $P$-value $<0.05$ for DEG selection.

\section{GO and KEGG pathway enrichment}

The Database for Annotation, Visualization, and Integrated Discovery (DAVID, Version 6.8, http://david.abcc. ncifcrf.gov/) can provide a comprehensive set of functional annotation tools for investigators to understand 
biological meaning behind a large list of genes ${ }^{24}$. Gene Ontology (GO) and Kyoto Encyclopedia of Genes and Genomes (KEGG) pathway enrichment analysis were performed using DAVID online tool, to analyze the DEGs at the functional level. $P<0.05$ was considered statistically significant.

\section{Real-time quantitative PCR}

Real-time quantitative PCR (qPCR) was used to detect the expression levels of mRNAs and miRNAs that were involved in this study. Total RNA was extracted using Trizol reagent (Invitrogen) according to the manufacturer's protocol. The primer sequences (AKAP12, PPARG2, miR-200b-3p, U6, and GAPDH) were listed in Supplementary Table S1. The conditions of qPCR amplification were as follows: the holding stage keeps $95^{\circ} \mathrm{C}$ for $5 \mathrm{~min}(1 \mathrm{cycle})$, the cycling stage holds $95^{\circ} \mathrm{C}$ for $15 \mathrm{~s}$ and $60^{\circ} \mathrm{C}$ for $45 \mathrm{~s}$ (40 cycles), the melt curve stage keeps $95^{\circ} \mathrm{C}$ for $15 \mathrm{~s}, 60^{\circ} \mathrm{C}$ for $1 \mathrm{~min}$, and $95^{\circ} \mathrm{C}$ for $15 \mathrm{~s}(1$ cycle). The whole amplifications were on Step OnePlus Real-Time PCR Systems (ABI, Life Technologies Corporation, USA). The $2^{-\Delta \Delta \mathrm{Ct}}$ method was applied to calculate the relative quantities of the target RNAs $^{25}$.

\section{Western blotting}

Total proteins of the tissues and cells were extracted using a protein extraction kit (Beyotime Biotechnology, Shanghai, China) according to the manufacturer's instructions. The extracted protein concentrations were detected using a bicinchoninic acid kit (Sigma-Aldrich, St. Louis, USA). The protein samples (each $40 \mu \mathrm{g}$ ) were separated using polyacrylamide gel electrophoresis $(10 \%$ concentration) and transferred to a polyvinylidene fluoride membrane. The membrane was then blocked with 5\% fat-free milk at room temperature for $1 \mathrm{~h}$ and incubated with primary antibody at $4{ }^{\circ} \mathrm{C}$ overnight. The next day, the membrane was washed with Tris-buffered Saline Tween 20 (TBST) for four times ( $3 \mathrm{~min} /$ time) and then incubated with the corresponding secondary antibody $(1: 2000$; catalog number 7056 or 7054, Cell Signaling Technology, Inc.) for $1 \mathrm{~h}$ at room temperature. Again, the membrane was washed with TBST for four times. The target bands were scanned and visualized using chemiluminescence method with Bio-Rad Gel Doc EZ imager (Life Science Research, CA, USA). Image J software (National Institutes of Health, MD) was applied to analyze the intensity of the target bands. The primary antibodies used were as follows: PPARG2 (1:800; catalog number sc-166731, Santa Cruz, CA), cyclinD1 (1:1000; catalog number sc-166731, Santa Cruz, CA), cyclinB1 (1:1000; catalog number 12231, Cell Signaling Technology, Inc.), p21 ${ }^{\text {Cip1 }}$ (1:1000; catalog number 2947, Cell Signaling Technology, Inc.), p27 ${ }^{\text {Kip1 }}$ (1:1000; catalog number 3686, Cell Signaling Technology, Inc.), Bcl-2 (1:1000; catalog number 4223,
Cell Signaling Technology, Inc.), AKT (1:1000; catalog number SAB4500797, Sigma-Aldrich, Saint Louis, MO, USA), p-AKT ( $1: 1000$; catalog number 05-802 R, SigmaAldrich, Saint Louis, MO, USA), DNA methyltransferase 3A (DNMT3A (1:1000; catalog number 32578, Cell Signaling Technology, Inc.), and DNMT3B (1:1000; catalog number 72335, Cell Signaling Technology, Inc.). $\beta$-Actin (1:1000; catalog number 3700, Cell Signaling Technology, Inc.) was used as an internal reference.

\section{Cell proliferation, colony formation, migration, and invasion}

For cell proliferation, cells $\left(3 \times 10^{3}\right.$ per well $)$ were seeded to a 96-well plate and cultured for $0,24,48,72$, and $96 \mathrm{~h}$. Then, $100 \mu \mathrm{l}$ of medium containing $10 \mu \mathrm{l}$ Cell Counting Kit-8 (CCK-8) reagent (Beyotime Biotechnology, Shanghai, China) was added to each well for incubation of another $2 \mathrm{~h}$ at $37^{\circ} \mathrm{C}$. The absorbance was then measured at $450 \mathrm{~nm}$ according to the manufacturer's instruction.

For 5-ethynyl-2'-deoxyuridine (EdU) incorporation assay, cells $\left(5 \times 10^{4}\right.$ per well) were cultured in 96-well plates at $37^{\circ} \mathrm{C}$ for $48 \mathrm{~h}$. Then, $100 \mu \mathrm{l}$ of medium containing $50 \mu \mathrm{M}$ EDU (catalog number C10310-1, RiboBio Biotechnology, Guangzhou, China) was added to each well for another $2 \mathrm{~h}$ at $37^{\circ} \mathrm{C}$ and fixed with $4 \%$ paraformaldehyde for $30 \mathrm{~min}$. Then, the fixed cells were permeabilized with $0.5 \%$ Triton X-100 for $10 \mathrm{~min}$. Finally, the cells were stained with Apollo® 567 and Hoechst33342, respectively.

For colony formation, cells were seeded to a six-well plate and cultured for 2 weeks. Then the cells were fixed with $4 \%$ paraformaldehyde for half an hour and stained with crystal violet (Sigma-C3886) for $10 \mathrm{~min}$, and then the colonies comprising over 50 cells were counted.

For migration of wound-healing assay, in brief, the cell monolayers of each well in a six-well plate were scratched using a $100 \mu \mathrm{l}$ pipette tip and photographed at 0 and $24 \mathrm{~h}$, respectively.

For cell invasion assay, the diluted Matrigel (catalog number 356234; BD Biosciences) was added to each Transwell upper chamber. Then, cells $\left(1 \times 10^{5}\right)$ that cultured in serum-free medium were added to the upper chambers and the complete medium was added to the lower chambers. After $36 \mathrm{~h}$, the cells were fixed with $4 \%$ paraformaldehyde for half an hour and stained with $0.5 \%$ crystal violet for $10 \mathrm{~min}$ at room temperature. Cells were counted under a microscope (Leica DM2500, Leica Microsystems, Inc.) at $\times 200$ magnification.

\section{Flow cytometry}

Cells $\left(1 \times 10^{6}\right.$ per well $)$ were seeded to a six-well plate. After $24 \mathrm{~h}$, cells were trypsinized and washed with precooled phosphate-buffered saline (PBS) and fixed with 
$70 \%$ ethyl alcohol at $4{ }^{\circ} \mathrm{C}$ overnight. Then, the cell suspension was incubated with propidium iodide $(0.5 \mathrm{mg} / \mathrm{ml})$ (Beyotime Biotech, Shanghai, China) for $15 \mathrm{~min}$. DNA content was analyzed using a flow cytometer (BD Biosciences, San Jose, CA).

\section{Tumor xenograft models}

The experiments were approved by the Research Ethics Committee of Nantong University according to Council on Animal Care Guidelines of Nantong University. A total of 12 $\mathrm{BALB} / \mathrm{c} 5$-week-old male nude mice were randomly divided into EV and PPARG2 groups (6 per group). EV or PPARG2transfected PC3 cells were injected subcutaneously into the flanks of the mice $\left(1 \times 10^{6}\right.$ cells $/ 100 \mu \mathrm{L}$ per flank). Tumor growth of mice was observed every 7 days, using a caliper for the tumor volumes. Thirty-five days later, all mice were killed and the tumors were weighted and photographed. Then, tumor tissues were used for hematoxylin and eosin (H\&E) staining and immunohistochemical analysis of Ki67 protein expression. The tumor volumes were measured and calculated using the following formula: volume $(V)=$ width $(W)^{2} \times$ length $(L) / 2$.

\section{Methylation-specific and bisulfite-sequencing PCR}

For methylation-specific PCR (MSP), the methylation status of $\mathrm{CPG}$ islands in AKAP12 gene promoter region were screened using MSP method initially in PCA cells. In brief, DNA samples that modified with bisulfite were extracted according to instructions of the manufacturer (Zymo Research, Orange, CA) first. Then, a total of $40 \mathrm{ng}$ of bisulfite-modified DNA was used for PCR amplification. After that, $10 \mu \mathrm{l}$ of the amplificated product was taken to analyze using Agarose gel electrophoresis. For interpretation of the MSP results, the methylated and unmethylated state is represented by the methylation (M) and unmethylation $(\mathrm{U})$ band, respectively. Occasionally, if the site is methylated partially, the two bands may appear.

Bisulfite-sequencing PCR (BSP) is a sequencing method to detect the methylation status of CpG islands. Briefly, DNA samples were treated with bisulfite and amplified by PCR. Then, the PCR products were purified using a TIANgel Midi Purification Kit (Tiangen Biotech, Beijing, China). After that, the purified products were cloned into a pGEM-T easy vector (Promega, Madison, WI, USA). Nine colonies were randomly chosen for plasmid DNA extraction using a Promega Spin Mini kit (Promega) and then sequenced by an ABI 3130 Genetic Analyzer (Applied Biosystems, Foster City, CA, USA).

\section{Dual luciferase reporter}

In brief, the $3^{\prime}$-untranslated region (3'-UTR) of DNMT3A/3B (wild type and mutant) were amplified first and cloned into the pmiR-RB-Report ${ }^{\mathrm{TM}}$ vector (Ribobio, Guangzhou, China), respectively. Then HEK 293T cells were co-transfected with pmiR-RB-Report ${ }^{\mathrm{TM}}$-WTDNMT3A/3B or pmiR-RB-Report ${ }^{\text {TM }}$-MUT-DNMT3A/ 3B and miR-200b-3p mimic/mimic control. After $48 \mathrm{~h}$, the Dual Luciferase Reporter Detection System (Promega, Madison, WI, USA) was used to detect luciferase activity. Firefly luciferase $(h L u c+)$ was the reporter gene and Renilla luciferase $(h R l u c)$ was the internal reference gene. The relative activity changes of $h L u c+/ h R l u c$ were detected to determine whether miRNAs could target $3^{\prime}$ UTR of the corresponding gene.

For AKAP12 gene promoter analysis, pGL3-Basic vector was selected for construction. Relative luciferase activity was detected by the Dual Luciferase Assay system (Promega). The phRL-TK vectors (Promega) were used as the internal reference.

\section{Chromatin immunoprecipitation}

Chromatin immunoprecipitation (ChIP) assay was performed using ChIP Enzymatic Chromatin IP Kit (Magnetic beads, Cell Signaling, Danvers, MA) according to the manufacturer's instructions. Briefly, the cells were crosslinked with formaldehyde of $1 \%$ final concentration first. Then, they were washed with pre-cold PBS and collected, followed by sonication crush. The solution complexes were immunoprecipitated using the anti-PPARG2 antibody (1:100; catalog number sc-166731, Santa Cruz, CA) or rabbit immunoglobulin G (IgG, negative control). After that, the immunoprecipitated complexes were collected using protein $\mathrm{G}$-agarose beads. The precipitates were eluted from the beads and the DNA-protein complexes were de-crosslinked at last. The DNA samples were recollected and used for PCR analysis. The PCR conditions were as follows: the holding stage keeps $95^{\circ} \mathrm{C}$ for $5 \mathrm{~min}$ ( 1 cycle), the cycling stage holds $95^{\circ} \mathrm{C}$ for $30 \mathrm{~s}, 55^{\circ} \mathrm{C}$ for $30 \mathrm{~s}$, and $72{ }^{\circ} \mathrm{C}$ for $30 \mathrm{~s}$ (35 cycles), and $72^{\circ} \mathrm{C}$ for 10 min (1 cycle). ChIP primers for detailed sequences were shown in Supplementary Table S1.

\section{Data analysis}

Statistical analysis was performed using the SPSS 17.0 statistical package (Chicago, IL, USA). Data were expressed as the mean \pm SD. The Student's $t$-test was used for comparison of two groups. Differences of multiple groups were compared using one-way analysis of variance (ANOVA). When ANOVA detects significant differences, the data were then compared using a Tukey's test as post hoc test. Correlation coefficient $(r)$ and $P$-values were calculated by Pearson's correlation analysis. $P<0.05$ was considered statistically significant.

\section{Results \\ PPARG2 is downregulated in PCA cell lines and tissues}

To determine the expression levels of PPARG2 in PCA cell lines and tissues, we first detected the relative mRNA 
expression levels in different PCA cell lines (LNCap, PC3, and DU145) and a normal prostate epithelial progenitor cell line NHPrE1 by qRT-PCR. We found it was significantly downregulated in the three PCA cell lines compared with the normal cell line (Fig. 1A). Then, the data extracted from The Cancer Genome Atlas (TCGA) showed that PPARG2 was downregulated in PCA tissue samples (496 cases) compared with the normal tissues (50 cases) (Fig. 1B).

To examine the protein expression level of PPARG2 in clinical PCA specimens, eight PCA tissues $(\mathrm{T})$ and eight prostate hyperplasia tissues $(\mathrm{N})$ were collected and analyzed. The results revealed lower PPARG2 protein expression was in the Tumor group than that in the Normal group (Fig. 1C, D). In addition, to obtain further insight into the function of PPARG2, the gene set enrichment analysis ${ }^{26}$ of TCGA profiles based on PPARG2 single gene expression was performed. The results indicated that PPARG2 expression levels were correlated negatively with cell proliferation by affecting genes in cell cycle regulation (Fig. 1E, F). Taken together, the above results reveal obviously that PPARG2 is downregulated in PCA.

\section{PPARG2 suppresses cell migration, colony formation,} invasion, and induces cell cycle arrest of PCA cells in vitro

To study the effects of PPARG2 on the biological behaviors, PC3 and LNCaP cell lines were selected as research represents of PCA cells in the following studies. The PPARG2-overexpressing lentivirus vector-GV358 containing the human PPARG2 wild-type full-length sequence (PPARG2) for gain-of-function and the lentivirus $\mathrm{EV}$ as a control were transfected respectively into the PCA cells. Results from wound-healing assays indicated that overexpression of PPARG2 suppressed cell migration significantly in PC3 and LNCaP cell lines (Fig. 2A, B). Overexpression of PPARG2 also significantly inhibited the colony numbers of PPARG2 group in the colony formation assay compared to those of the EV group (Fig. 2C, D). At the same time, Transwell cell invasion tests showed that the cells' abilities of the PPARG2 group to migrate and penetrate Matrigel was significantly reduced compared with those of the EV group (Fig. 2E, F). These results indicate that PPARG2 may inhibit the proliferation and tumorigenicity of PCA cells.

To further evaluate the potential suppressing effects of PPARG2 on cell proliferation, CCK-8 assay was performed in 1, 2, 3, and 4 days after Lv-PPARG2 and Lv-EV transfection. Compared with the EV group, a significant decrease of cell viability was detected in PC3 and LNCaP cells in the PPARG2 group (Fig. 3A). Then, EdU retention assays were performed to assess the inhibiting effect of PPARG2 on DNA replication. Following transfection with
Lv-PPARG2, the percentage of EdU-positive cells was decreased significantly in PC3 and LNCaP cells compared to EV group (Fig. 3B, C).

Moreover, to identify the mechanism through which PPARG2 overexpression inhibits the proliferation of PCA cells, we checked the cell cycle distribution in PC3 and LNCaP cells transfected with Lv-PPARG2 or Lv-EV using flow cytometry (Fig. 3D, E). The results showed cell cycle of G1 arrest that the cell populations in the G1 phase of the cell cycle increased significantly in the PPARG2 group compared with EV-transfected controls of PC3 and LNCaP cells. However, the cell populations were reduced in the S stage of the cell cycle compared to the EV controls in the two cell lines. The results indicated that progression of G1-S cell cycle was inhibited by PPARG2 overexpression in PC3 and LNCaP cells. In addition, the protein expression levels of cyclinD1, cyclinB1, p21 ${ }^{\text {Cip1 }}$ and $\mathrm{p} 27^{\mathrm{Kip} 1}, \mathrm{Bcl}-2, \mathrm{p}-\mathrm{AKT}$, and AKT were also analyzed between the two groups in PC3 and LNCaP cells (Fig. 3F-H). Western blotting analysis revealed that cyclinD1, Bcl-2, and p-AKT were decreased significantly in PPARG2-transfected cells. Conversely, cell cycle inhibitors $\mathrm{p} 21^{\mathrm{Cip} 1}$ and $\mathrm{p} 27^{\mathrm{Kip} 1}$ were upregulated in PPARG2transfected cells. Moreover, altering expression of PPARG2 in the two groups had no effect on the protein expression of AKT and cyclinB1.

\section{PPARG2 inhibits tumor growth in vivo}

To further study the anticancer effects of PPARG2 on PCA progression in vivo, xenograft models were established via injection subcutaneously of PC3 cells treated with PPARG2 or EV into BALB/c nude mice. Compared with the EV group, the xenograft tumor volumes and weights in the PPARG2 group were all markedly decreased (Fig. 4A-C). Moreover, H\&E and proliferating cell-associated antigen Ki67 staining were performed to study the proliferation level of the subcutaneous tumors. H\&E-staining results showed the nuclei in both groups were large and deeply stained, whereas Ki67-staining results in tumor xenografts indicated that the Ki67positive rate was decreased markedly in the PPARG2 group, suggesting that PPARG2 can inhibit tumorigenicity of PCA cells in vivo (Fig. 4D, E).

\section{PPARG2-mediated induction of AKAP12 mRNA upregulation in vitro}

To further explore the potential mechanisms of PPARG2-suppressing cell proliferation of PCA, gene expression microarray was performed using PC3 cells between overexpressing PPARG2 cDNA (PPARG2) and $E V$. The clustering heat map of differentially expressed genes between sample groups was shown in Supplementary Fig. S1A. Then, differentially expressed genes were screened to meet fold change $>1$ and $P$-value $<0.05$ 
A

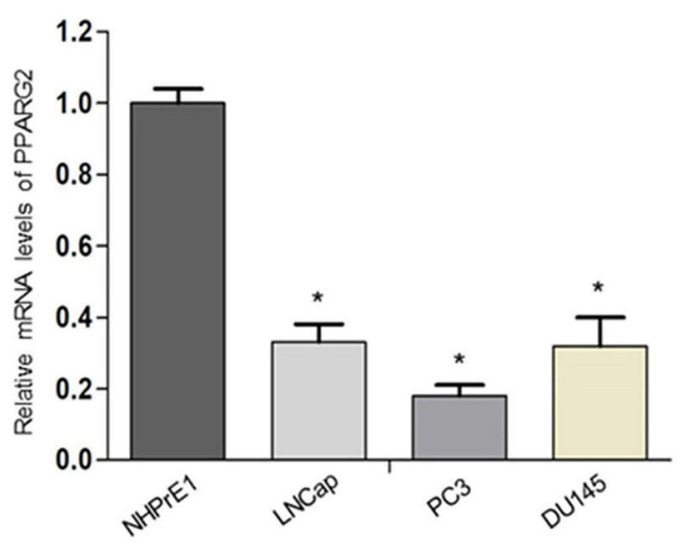

C
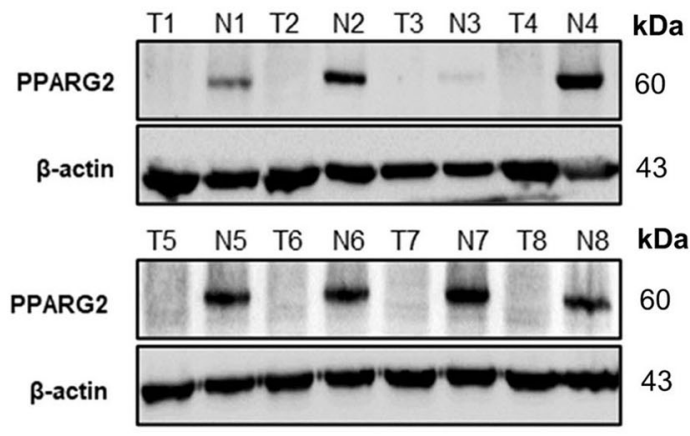

E

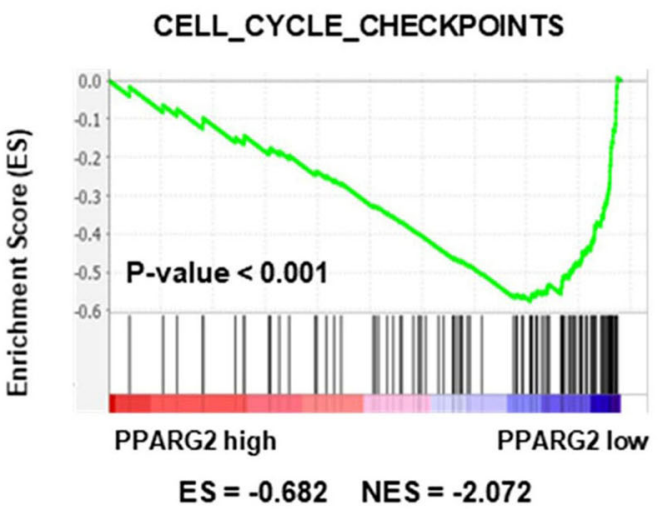

B

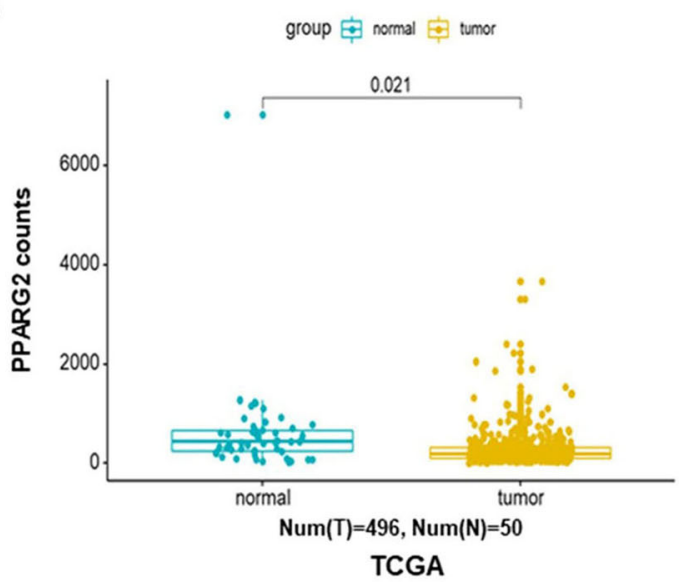

D

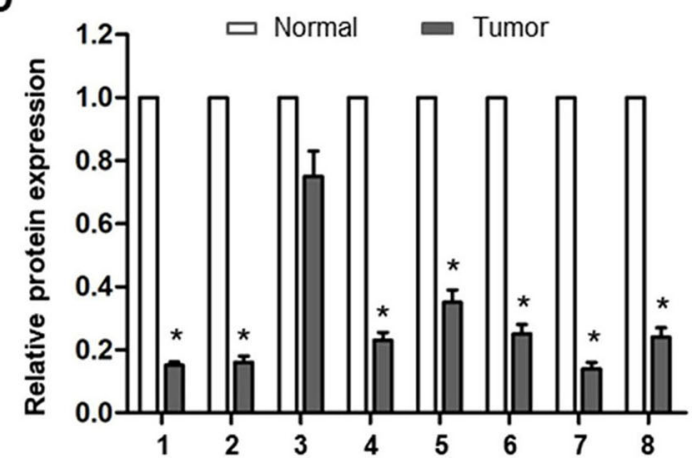

$\mathbf{F}$

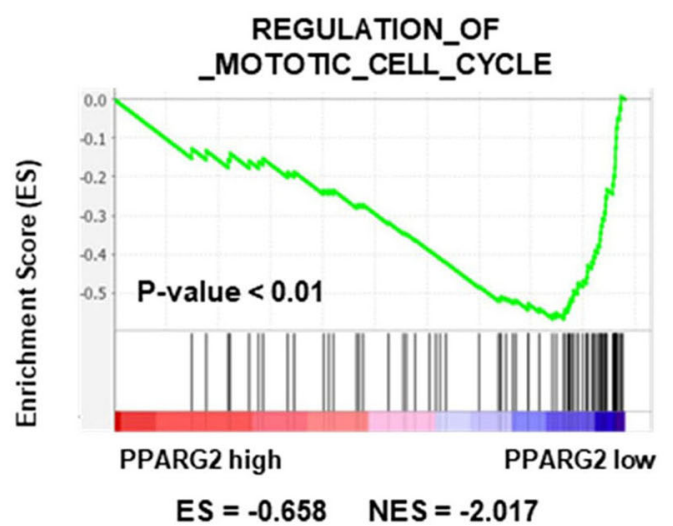

Fig. 1 PPARG2 is downregulated in PCA, negatively correlated with cell cycle-activated gene signatures. A Relative mRNA expression levels were analyzed in different PCA cell lines and a normal prostate epithelial progenitor cell line NHPrE1 by qRT-PCR. ${ }^{*} P<0.05$ vs. normal group. B Expression of PPARG2 in 496 PCA tissues (tumor) compared with 50 normal tissues (normal) in the TCGA profile. $P=0.021$. C, D Western blotting analyses of relative PPARG2 protein expression in eight PCA tissues (T) and eight prostate hyperplasia tissues $(N)$. ${ }^{*}<<0.05$ vs. normal group. E, F GSEA plot showing the PPARG2 expression level correlated negatively with cell cycle-activated gene signatures (CELL_CYCLE_CHECKPOINTS, REGULATION_OF_MITOTIC_CELL_CYCLE).

between the two groups, of which 716 genes were upregulated and 822 genes were downregulated (Supplementary Fig. S1B). From the upregulated gene set, we screened the AKAP12 gene (Fig. 5A) and the expression levels were significantly lower in PCA tissues than those in normal ones from the TCGA database (Fig. 5B). Moreover, correlation analysis from Gene Expression Profiling Interactive Analysis (GEPIA) (Fig. 5C) and TCGA (Fig. 5D) database indicated that the expressions between the two genes was positively correlated significantly. In addition, PPARG2 also showed a positive correlation in mRNA expression with AKAP12 in most cancer and 


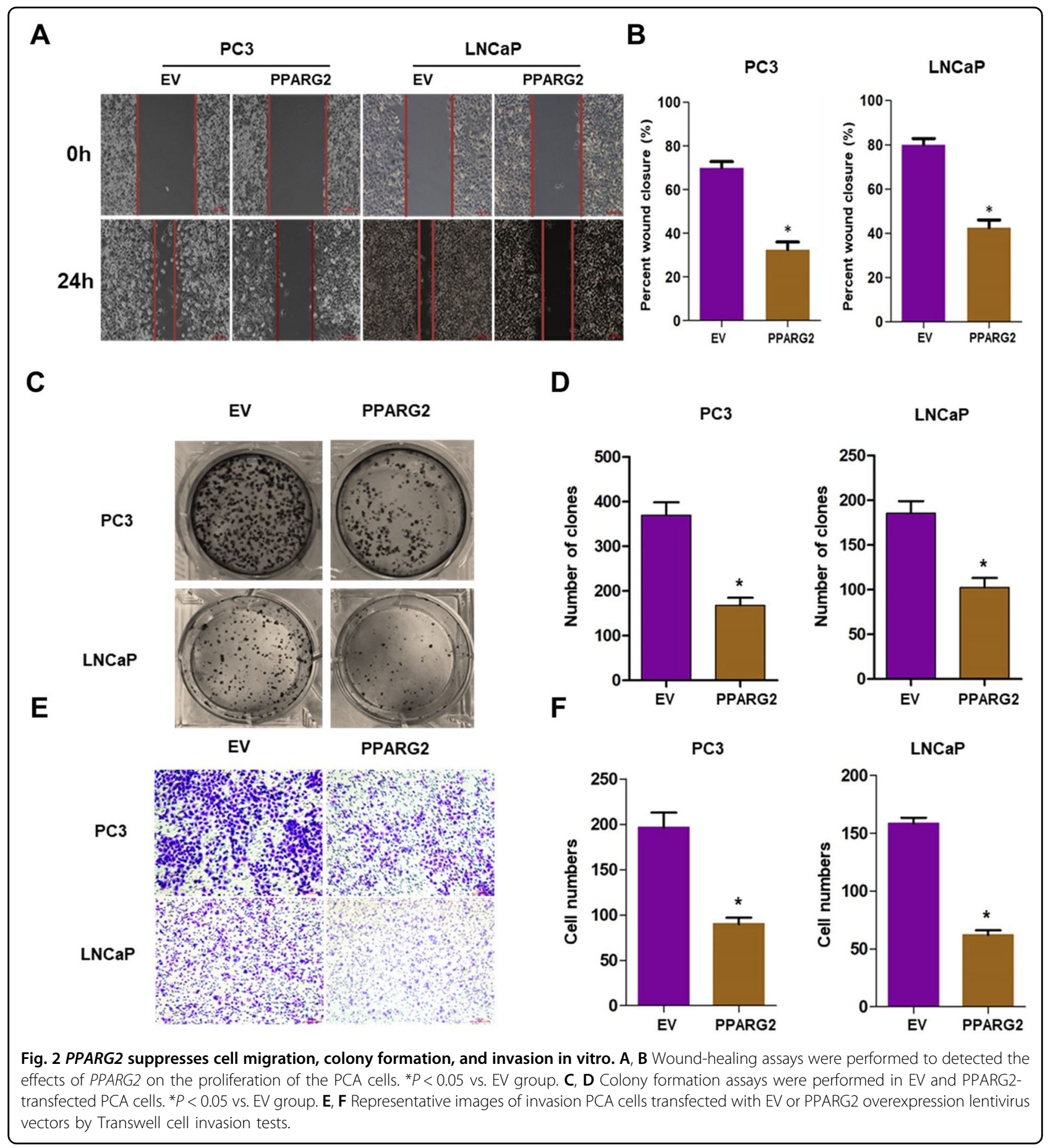

normal tissues or cell lines (Fig. 5E, F). Then, functional analysis of GO enrichment and KEGG pathway were performed to investigate the functions and processes of the target gene set using the online software DAVID. The results revealed that GO enrichment involved in (i) biological process (BP) (Supplementary Fig. S2A), such as positive regulation of transcription, positive regulation of apoptotic process, and DNA methylation; (ii) involved in molecular function (Supplementary Fig. S2B), such as protein binding, TF binding, and poly(A) RNA binding; and (iii) involved in cellular component (Supplementary Fig. S2C), such as nucleoplasm, nucleus, and cytoplasm. The KEGG pathway analysis suggested that the differentially expressed genes of PC3-PPARG2 cells were involved in cell cycle, 


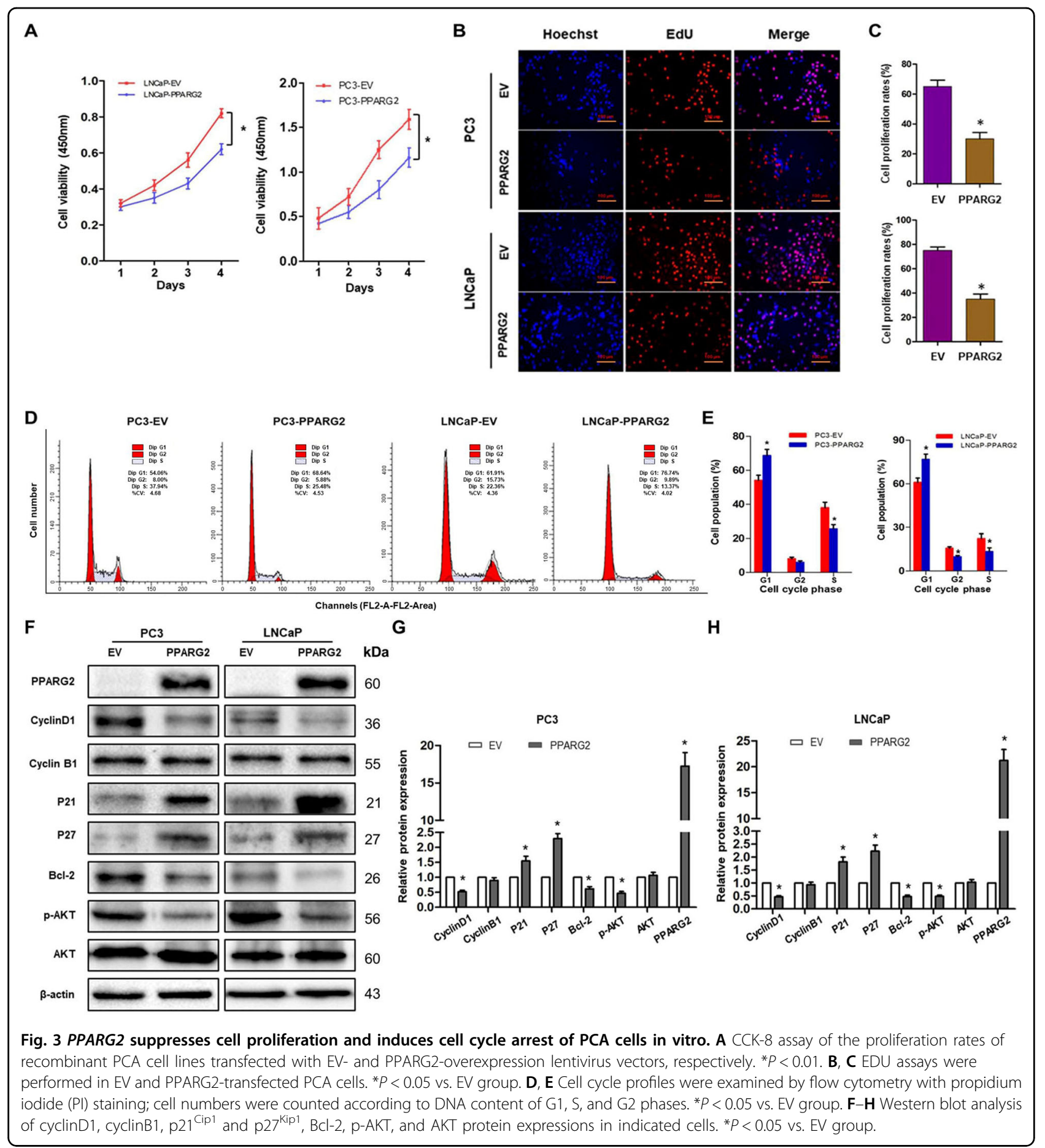

PI3K-Akt signaling pathway, and transcriptional misregulation in cancer, etc. (Supplementary Fig. S2D).

\section{Upregulated PPARG2 induces demethylation of the AKAP12 promoter region in vitro}

From the above analysis results of BP, which is under GO classification, we found the target gene set were involved in DNA methylation. Then, expression levels of the three DNA methyltransferase (DNMT1, DNMT3A, and $D N M T 3 B$ ) were extracted from the microarray data. The results indicated that the expression levels of $D N M T 3 A$ and DNMT3B were all downregulated markedly in the PPARG2 group compared with those of the EV group (Supplementary Fig. S3B, C), whereas 


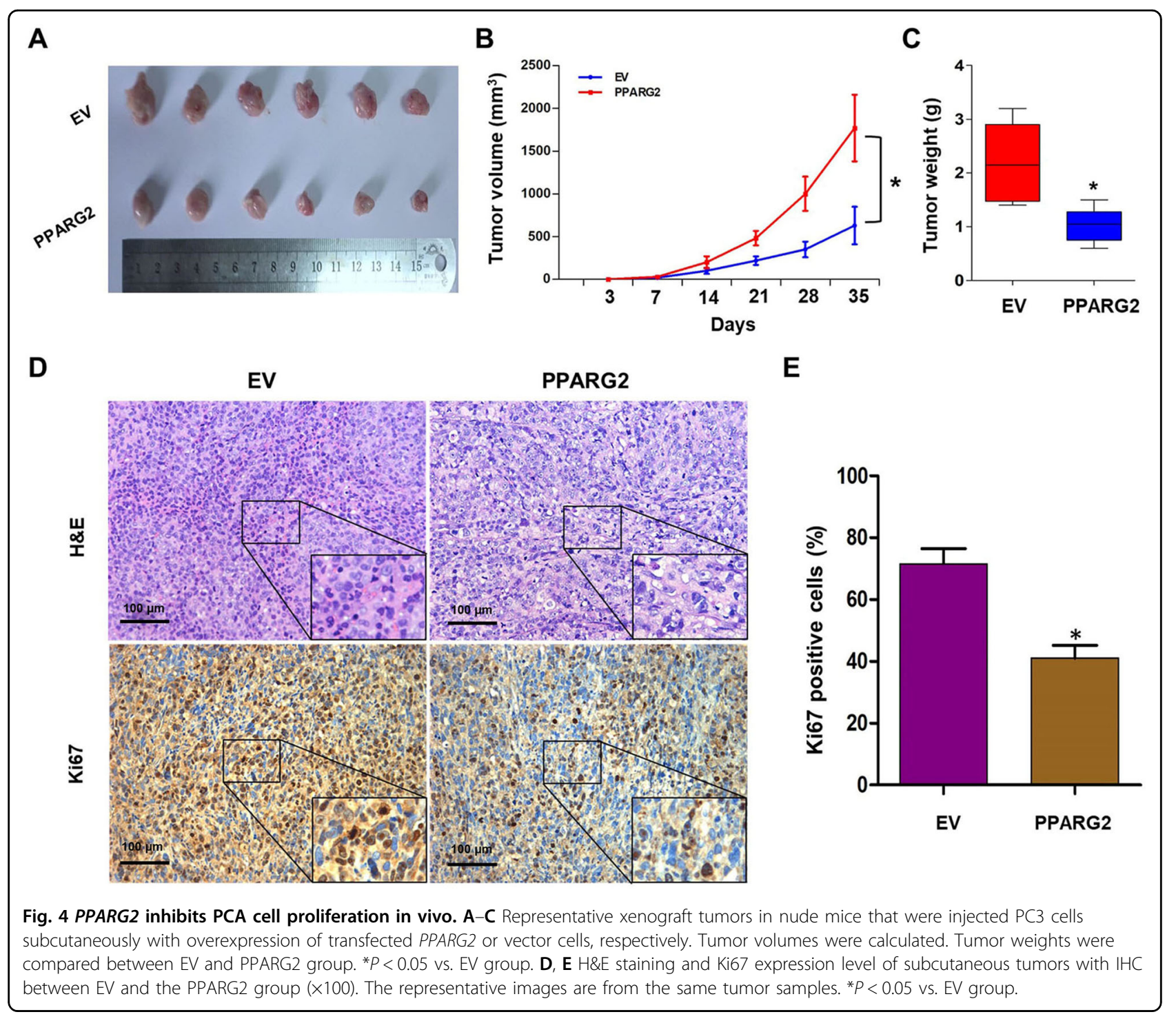

DNMT1 expression level showed a nonsignificant upregulation in the PPARG2-treated group (Supplementary Fig. S3A).

To further investigate the intrinsic mechanism of PPARG2-induced upregulation of AKAP12 and downregulation of $D N M T 3 A / 3 B$, we then conducted the following experiments to clarify it from the perspective of epigenetics. We first scanned the AKAP12 promoter for potential regions of DNA methylation and found obvious CpG islands existed in the promoter region near the transcription start site (TSS) (Fig. 6A). Then, the CpG island demethylation statuses were detected between the PPARG2 and EV group by using MSP. The demethylation levels of the AKAP12 promoter region were significantly increased in the PPARG2 group (Fig. 6B), which could be the result of downregulated DNMT3A/3B expression in the PPARG2 group. Moreover, to obtain further demethylation status details of specific CpG sites near the promoter region of AKAP12 between the PPARG2 and EV group, a $294 \mathrm{bp}$ length of PCR product ( -315 to $-22 \mathrm{bp}$ ) was analyzed following sodium bisulfite treatment using BSP method (Fig. 6C). The results revealed lower methylation frequencies in the PPARG2 group than those in the EV group (Fig. 6D, E).

In addition, to determine which $\mathrm{CpG}$ sites were responsible for the demethylation-related activation of the AKAP12 gene under the condition of PPARG2 upregulation in PCA cells, two AKAP12 gene promoter regions (PGL3-180 and PGL3-315) were constructed and treated with $S s s I$ methylase in vitro before being transfected into the PC3 cell line (Fig. 6F). In comparison with the untreated promoter construct, the SssI methylase-treated construct showed a significant suppression of promoter activity. Although there were no significant differences in 


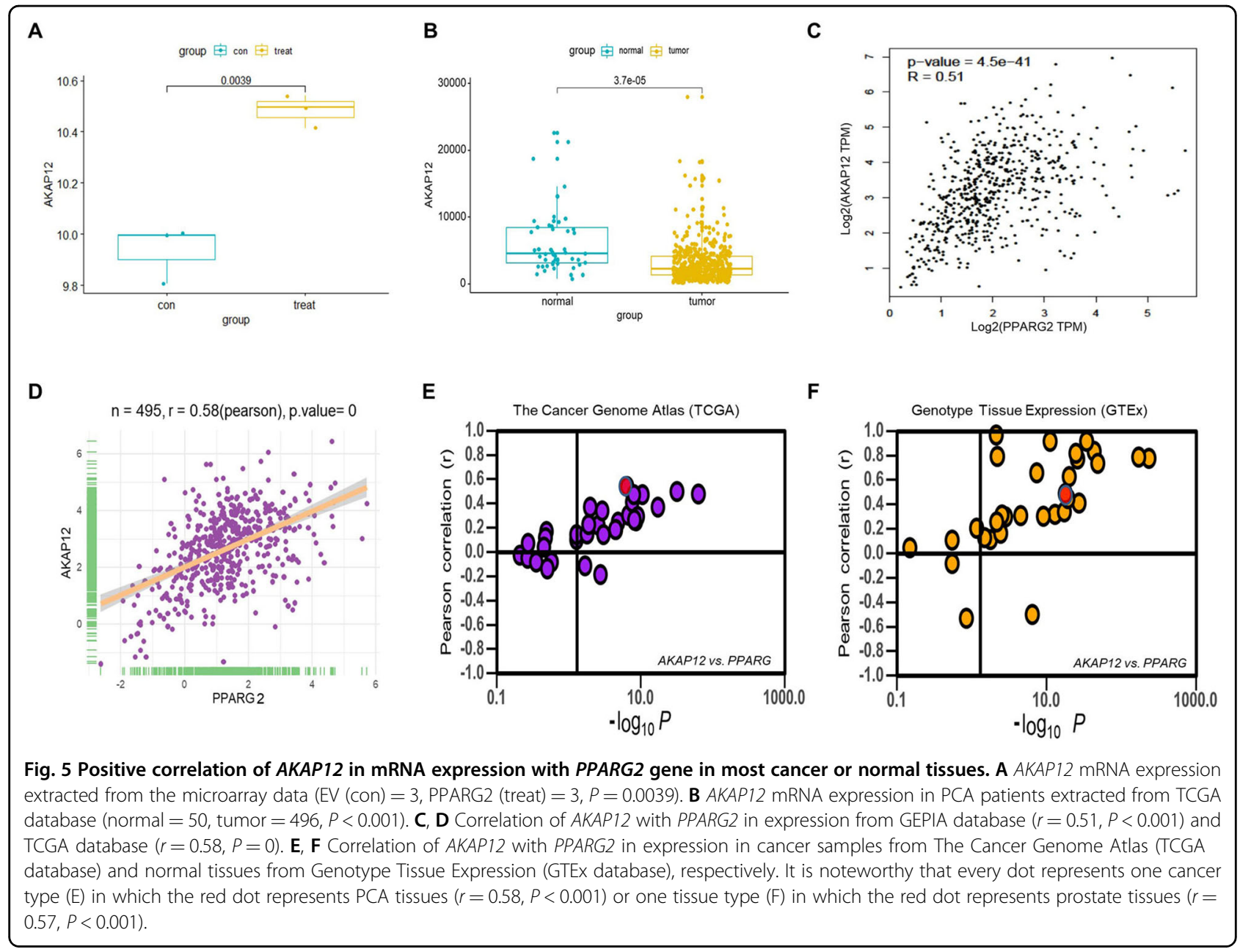

the promoter activity between the region of PGL3-180 and PGL3-315 with or without SssI methylase treatment, it indicated that the promoter region at $-180 \mathrm{bp}$ may play an important role in regulating $A K A P 12$ gene transcription. Finally, relative $A K A P 12$ mRNA expression levels were detected after PC3 cells were treated with different concentrations $(0,1,2$, and $3 \mu \mathrm{M})$ of DNMT inhibitor 5Aza-2'-deoxycytidine (5-Aza-dc). The detection results showed a 5-Aza-dc dose-dependent significant upregulation of AKAP12 mRNA expression levels (Fig. 6G), which indicated that methylation statuses of the related region in AKAP12 promoter were involved in regulation of its expression.

\section{Upregulation of miR-200b-3p regulates expression of the downstream target genes DNMT3A/3B in PPARG2- overexpressed PCA cells}

To further explore the mechanism of upregulated PPARG2 causing downregulation of DNMT3A/3B, one of the possibilities we thought was that miRNAs may be involved in the regulatory function of the genes. Then,
miRNA-seq was performed between PPARG2 and EV group of the PC3 cell line. The clustering heat map of differentially expressed miRNAs between sample groups were shown in Supplementary Fig. S4A. Differentially expressed miRNAs were screened, and 18 miRNAs were upregulated and 44 miRNAs were downregulated (Supplementary Fig. S4B). From the upregulated 18 miRNAs, we identified miR-200b-3p as the study target, which was upregulated significantly in the PPARG2 group compared with the EV group extracted from miRNA-seq results (Fig. 7A), and it has been then confirmed by experimental verification (Fig. 7B).

The next step, the regulatory relationship between miR200b-3p and DNMT3A/3B, was confirmed via experimental verification. Bioinformatics revealed that $D N M T 3 A / 3 B \quad 3^{\prime}$-region contained one conserved target site of miR-200b-3p, respectively (Fig. $7 \mathrm{C}$ ). To evaluate this prediction, the wild-type $3^{\prime}$-UTR sequence of $D N M T 3 A / 3 B$ (wild type) or its mutant sequence (Mut) (Fig. 7D, E) was subcloned into the pmiR luciferase reporter and then co-transfected with miR-CON or miR- 


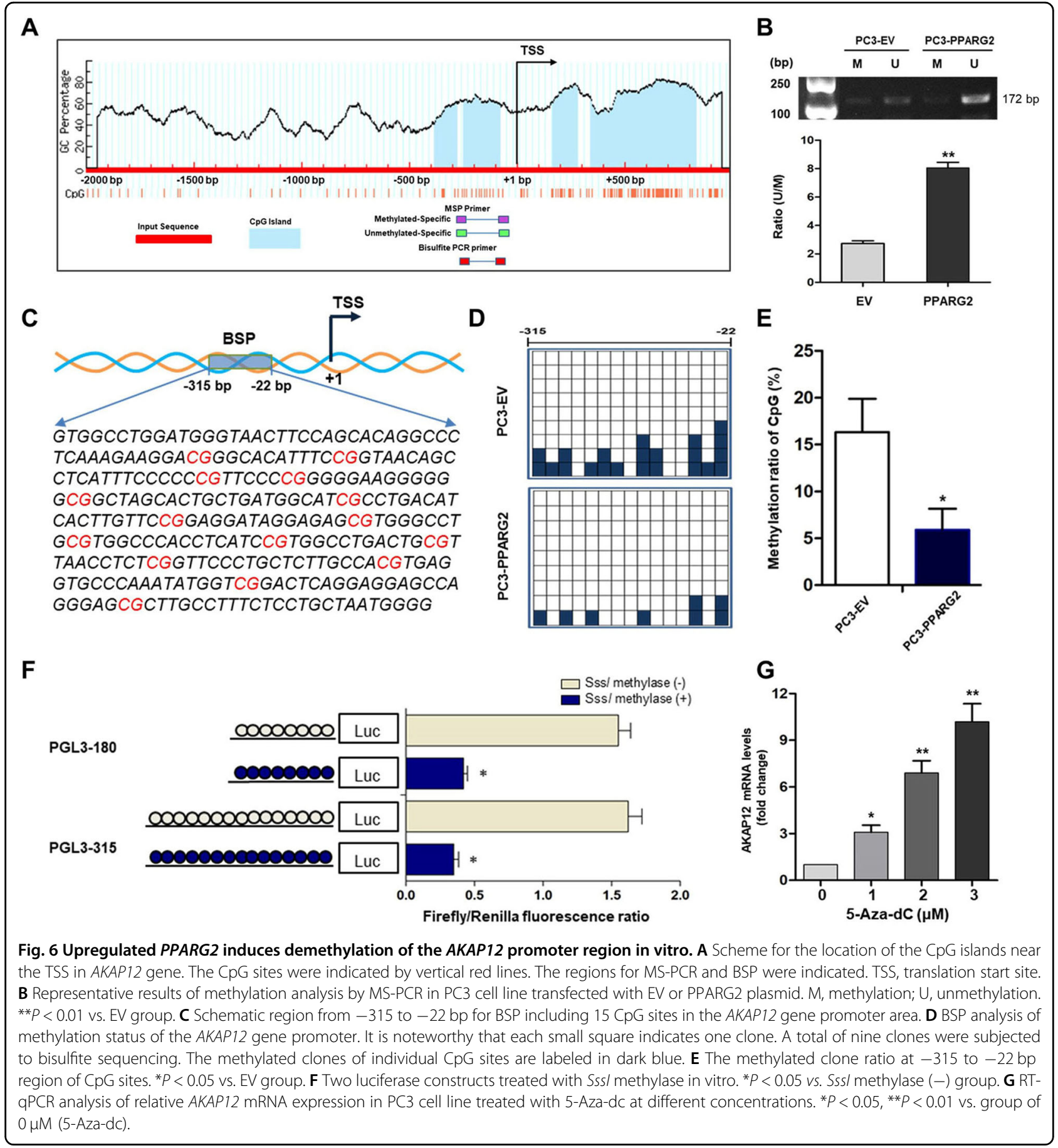

200b-3p mimic into 293T cells. The results indicated that the relative luciferase activity of the pmiR wild type was decreased significantly by $45.0 \%$ (Fig. 7D) and 51.3\% (Fig. $7 \mathrm{E})$, respectively, when miR-200b-3p mimic was cotransfected into the cells. However, no differences of relative luciferase activity of pmiR-Mut showed when cotransfected with miR-CON or miR-200b-3p mimic into PC3 cells.
Moreover, the qPCR-detected results verified that relative miR-200b-3p expression levels showed significant upregulation or downregulation in miR-200b-3p mimic or miR-200b-3p inhibitor-treated PC3 cells (Fig. 7F). Finally, we examined DNMT3A/3B expression in PC3 cells by western blotting. As expected, miR-200b-3p mimic triggered a significant suppressing effect on DNMT3A/3B protein expression. However, the protein expression levels 


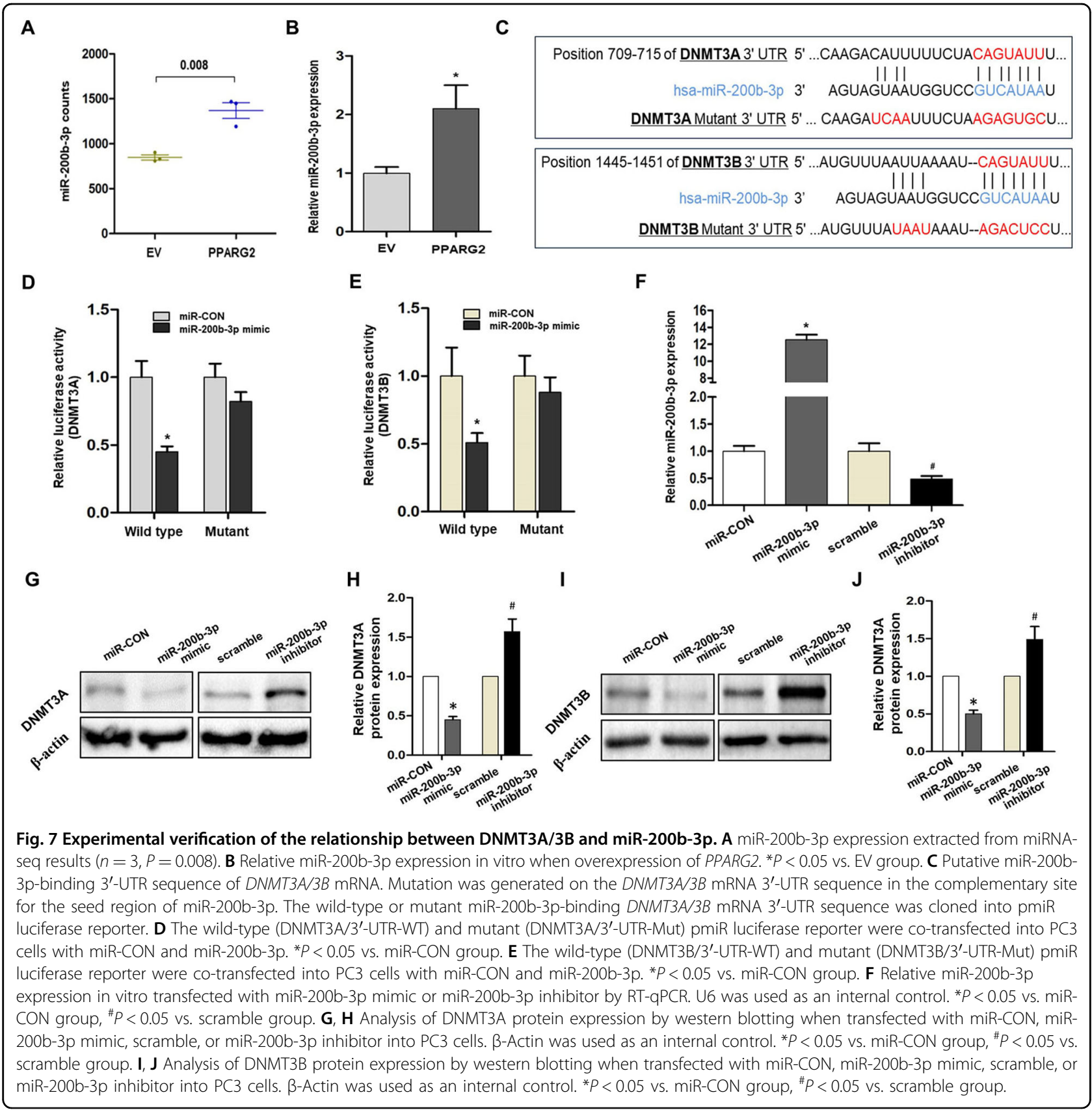

of DNMT3A/3B were regained in miR-200b-3p inhibitortreated PC3 cells (Fig. 7G-J). The results further confirmed the regulatory effect of miR-200b-3p on DNMT3A/3B mRNA.

\section{Enhanced binding of PPARG2 to AKAP12 promoter region in PPARG2-overexpressed PC3 cells}

To further explore the mechanism by which PPARG2 induces the upregulation of $A K A P 12$, we thought of PPARG2, which, as a kind of TF, may be involved in binding to the promoter region of $A K A P 12$. We predicted the potential TF-binding site near the AKAP12 promoter region using the online software JASPAR (http://jaspar. genereg.net/) and found two potential PPARG2-binding sites (site $1,-1540$ to $-1521 \mathrm{bp}$ and site $2,-160$ to $-141 \mathrm{bp}$ ) (Fig. 8A). Then ChIP assay was performed to verify the prediction. The results revealed that PPARG2 could bind to the two binding sites. Meanwhile, enhanced binding of PPARG2 can be found in PPARG2-overexpressed PC3 cells (Fig. 8B). 


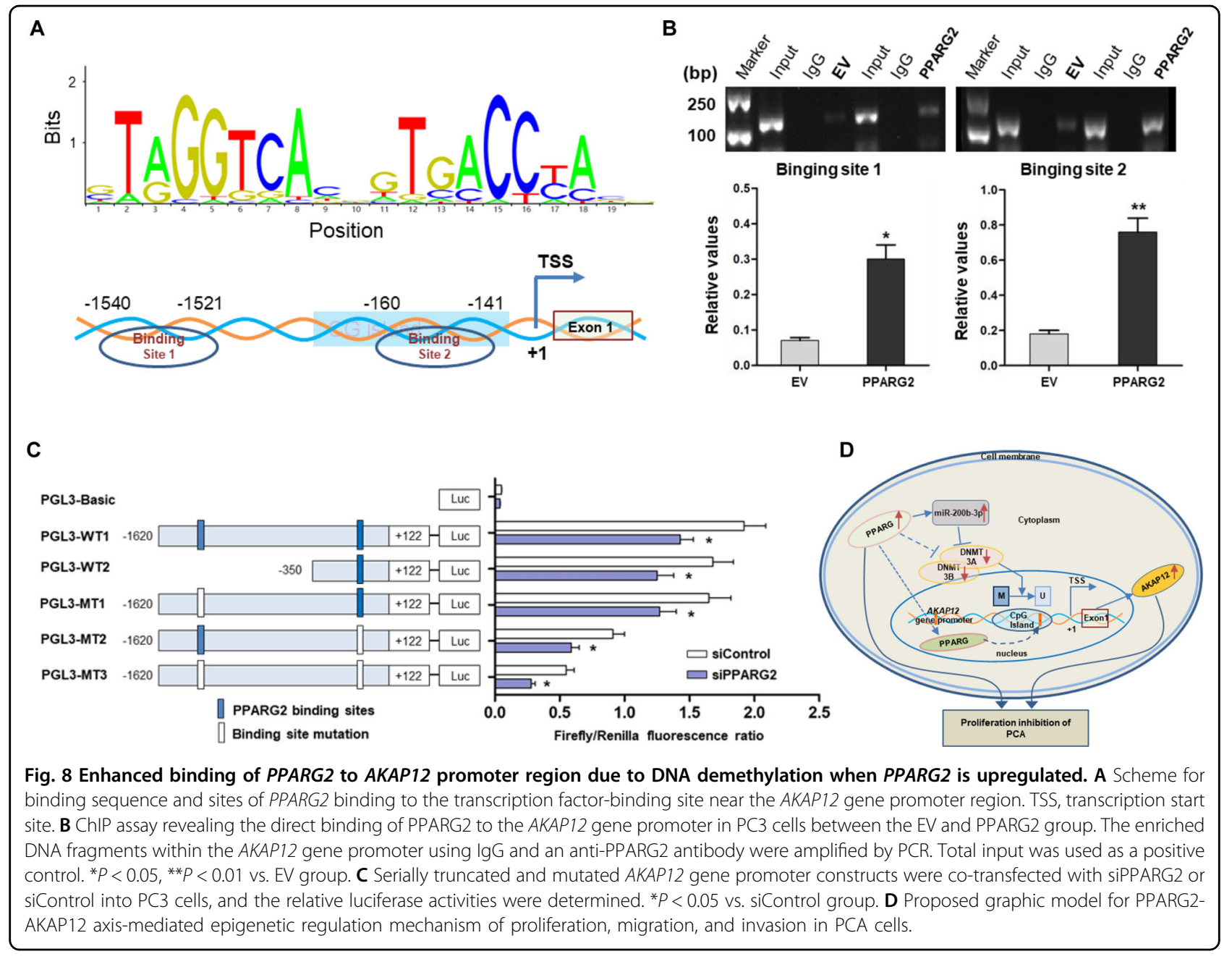

Moreover, to further verify the efficiency of the binding sites from ChIP assay results, PGL3 plasmids containing serially truncated and mutated AKAP12 gene promoter were constructed and co-transfected with siControl or siPPARG2 into $293 \mathrm{~T}$ cells, to determine the most effective functional binging site that caused PPARG2-regulated AKAP12 gene promoter activation (Fig. 8C). Luciferase reporter activity detection results showed a significant reduction of promoter activity in the cells that cotransfected with siPPARG2 of both PGL3-WT and PGL3-MT groups. At the same time, the reduced promoter activity caused by sequence truncation was not very obvious. From the luciferase results of serially mutated AKAP12 gene promoters (pGL3-MT1, pGL3-MT2,and pGL3-MT3), a more significant reduction in AKAP12 gene promoter activity was found in pGL3-MT2 (site 1, -160 to $-141 \mathrm{bp}$ ) or pGL3-MT3 (two sites mutation simultaneously). The results suggested that the PPARG2binding site 2 may play a more important role in $A K A P 12$ transcriptional activation.

\section{Discussion}

Peroxisome proliferator-activated receptors (PPARs) are involved in many diseases such as cancer ${ }^{27,28}$, diabetes $^{29}$, and inflammation ${ }^{30}$. Studies have revealed that PPARG acts as a tumor suppressor and plays an important role in tumorigenesis ${ }^{31,32}$. Additional studies showed PPARG as an oncogene in the development of tumors $^{33,34}$. Regarding the dual role of PPARG gene played in the occurrence and development of tumors, we believe it may be related to multiple factors involved in tumor types and tumor progression in a specific environment. In this study, our research target PPARG2, one of the PPARG protein isoforms, was found to be downregulated in PCA and inhibited cell migration, colony formation, invasion, and induced cell cycle arrest of PCA cells in vitro. Moreover, PPARG2 overexpression modulates the activation of the Akt signaling pathway, as well as inhibit tumor growth in vivo. This is our first report to elaborate the functional significance of PPARG2 expression in human PCA and experiment 
results indicated that PPARG2 functioned as a tumor suppressor and inhibits PCA malignant progression. Thus, PPARG2 holds great prospects as a new therapeutic target for PCA.

As TFs, most of members of PPARs can bind to the specific sites of the target gene promoter region to regulate their expression ${ }^{35}$. Therefore, for the next step for mechanism exploration, we screened differentially expressed genes via gene expression microarray from the PPARG2 and EV groups in vitro first. We found that AKAP12 mRNA was upregulated, mediated by PPARG2 overexpression. Reports have revealed that AKAP12 is a protein kinase $C$ substrate and a potential tumor suppressor in many types of cancers including hepatocellular carcinoma $(\mathrm{HCC})^{36}$, colorectal cancer ${ }^{37}$, and breast cancers $^{38}$. Moreover, interestingly, GO enrichment results indicated PPARG2 overexpression involved in DNA methylation of BP. Accordingly, we then associated AKAP12 gene with DNA methylation. We found that obvious $\mathrm{CpG}$ islands existed in its promoter region near the TSS and confirmed that upregulated PPARG2 induced demethylation of the AKAP12 promoter region via MSP and BSP experiments. Moreover, relative AKAP12 mRNA expression levels increased in a 5-Aza-dc dose-dependent concentration, which indicated that methylation statuses of AKAP12 promoter were involved in regulation of its expression. The above results indicated PPARG2AKAP12 axis-mediated epigenetic regulatory network may play a role in PCA.

Enzymes that catalyze CpG methylation in DNA sequence include DNA methyltransferase 1 (DNMT1), DNMT3A, and DNMT3B. These DNA methyltransferases are essential for mammalian tissue development and homeostasis, and also related to human developmental disorders and cancer, which supports that DNA methylation plays a key role in the specification and maintenance of cell fate ${ }^{39,40}$. In this study, we found that expression levels of DNMT3A and DNMT3B were all downregulated markedly in the PPARG2 group compared with those in EV the group. This data coincided with the upregulation of AKAP12 due to its promoter demethylation.

The next step, we explored the potential mechanism of $D N M T 3 A / 3 B$ downregulation induced by PPARG2 overexpression in PCA. It may be two ways: a direct regulation or an indirect effect through a mediator. Regarding the possible indirect influence mechanism, miRNAs could be suitable mediators, which belong to noncoding RNAs family. They mainly cause mRNA translational repression or degradation by targeting to the $3^{\prime}$-UTR of mRNAs ${ }^{41}$. Thus, we screened out miR-200b-3p using miRNA-seq and the DNMT3A/3B 3'-UTR region contains conserved target sites of miR-200b-3p. Studies have shown that miR200b-3p was downregulated in androgen-independent $\mathrm{PCA}^{42}, \mathrm{HCC}^{43}$, and glioma ${ }^{44}$. Our experiments confirmed the regulatory relationship between miR-200b-3p and $D N M T 3 A / 3 B$. The data indicated that miR-200b-3p participated indirectly in PPARG2-AKAP12 axis-mediated epigenetic regulatory network in PCA.

In addition, the ChIP results demonstrated the enhanced binding of PPARG2 to AKAP12 gene promoter in PPARG2-overexpressed PC3 cells and the sequential deletion and mutation analyses revealed that binding site $2(-160$ to $-141 \mathrm{bp})$ may play a more important role in the AKAP12 gene promoter activity. Coincidentally, the binding site 2 is exactly where CG island is located. It is obvious that increased affinity of PPARG2 to the specific binding site is due to the CG island demethylation. The data revealed that DNA demethylation enhanced the binding of PPARG2 to the AKAP12 gene promoter and participated in regulation of the AKAP12 gene expression.

In summary, the present work elucidates a PPARG2AKAP12 axis-mediated epigenetic regulatory network in PCA (Fig. 8D). PPARG2 acts as a tumor suppressor in suppressing malignancy of PCA cells in vitro and in vivo. As a transcriptional factor, PPARG2 overexpression induces the increased expression level of miR-200b-3p, which targeted $3^{\prime}$-UTR of the downstream targets $D N M T 3 A / 3 B$, facilitates interaction with demethylated AKAP12 gene promoter and suppresses cell proliferation in PCA via AKT signaling pathway. Of course, we recognize that upregulated PPARG2 potentially regulate a cluster of miRNAs, while one miRNA can target lots of genes, and involves in cross-talk pathways in the complex and elaborate epigenetic regulatory network in PCA. Therefore, we need to make more efforts to better explore the sophisticated mechanisms of PPARG2 regulation in the progression of PCA. In brief, our findings provided the first evidence for a novel PPARG2-AKAP12 axis-mediated epigenetic regulatory network. The study identified a molecular mechanism involving an epigenetic modification that could be possibly targeted as an antitumoral strategy against prostate PCA.

\section{Acknowledgements \\ We thank Dr. Changlai Zhu for flow cytometer operation.}

\section{Author details}

'Department of Pathology, Key Laboratory of Microenvironment and Translational Cancer Research, Medical School of Nantong University, Nantong 226001 Jiangsu, China. ${ }^{2}$ Department of Laboratory Medicine, Affiliated Hospital of Nantong University, Nantong 226001 Jiangsu, China. ${ }^{3}$ Basic Medical Research Centre in Medical College of Nantong University, Nantong 226001 Jiangsu, China

\section{Author contributions}

F.D. and F.L. concepted and designed the study. F.L., T.L., and D.L. performed experiments and collated the data. T.L. and Y.Z. contributed to conducting in vivo experiments. F.L. and C.Z. contributed to data analysis and manuscript drafting. All authors have read and approved the final submitted manuscript. 


\section{Funding}

This study was supported by the National Natural Science Foundation of China (Grant number 81874171), Postgraduate Research \& Practice Innovation Program of Jiangsu Province (Grant number KYCX20_2797), Nantong Science and Technology Project (numbers JC2020027, MS12018066, and MSZ19216).

\section{Ethics statement}

The protocol was approved by the Ethics Committee of the Affiliated Hospital of Nantong University. All patients obtained informed consent to participate in this study.

\section{Consent for publication}

All the authors agree to publish this paper.

\section{Conflict of interest}

The authors declare no competing interests.

\section{Publisher's note}

Springer Nature remains neutral with regard to jurisdictional claims in published maps and institutional affiliations.

Supplementary information The online version contains supplementary material available at https://doi.org/10.1038/s41419-021-03820-7.

Received: 18 December 2020 Revised: 11 May 2021 Accepted: 11 May 2021 Published online: 22 May 2021

\section{References}

1. Siegel, R. L., Miller, K. D. \& Jemal, A. Cancer statistics, 2018. CA Cancer J. Clin. 68, 7-30 (2018)

2. Roth, J. A., Gulati, R., Gore, J. L., Cooperberg, M. R. \& Etzioni, R. Economic analysis of specific antigen screening and selective treatment strategies. JAMA Oncol. 2, 890-898 (2016)

3. $\mathrm{Xu}, \mathrm{Y}$. et al. Family history is significantly associated with prostate cancer and its early onset in Chinese population. Prostate 79, 1762-1766 (2019).

4. Fenton, J. J. et al. Prostate-specific antigen-based screening for prostate cancer: evidence report and systematic review for the US preventive services task force. JAMA 319, 1914-1931 (2018).

5. Eldred-Evans, D. et al. Rethinking prostate cancer screening: could MRI be an alternative screening test? Nat. Rev. Urol. 17, 526-539 (2020).

6. Thurairaja, R., McFarlane, J., Traill, Z. \& Persad, R. State-of-the-art approaches to detecting early bone metastasis in prostate cancer. Bju. Int. 94, 268-271 (2004).

7. Litwin, M. S. \& Tan, H. J. The diagnosis and treatment of prostate cancer: a review. JAMA 317, 2532-2542 (2017).

8. Dubois, V., Eeckhoute, J., Lefebvre, P. \& Staels, B. Distinct but complementary contributions of PPAR isotypes to energy homeostasis. J. Clin. Invest. 127, 1202-1214 (2017).

9. Jiang, M., Shappell, S. B. \& Hayward, S. W. Approaches to understanding the importance and clinical implications of peroxisome proliferator-activated receptor gamma (PPARgamma) signaling in prostate cancer. J. Cell. Biochem. 91, 513-527 (2004)

10. Jiang, M., Strand, D. W., Franco, O. E., Clark, P. E. \& Hayward, S. W. PPARgamma: a molecular link between systemic metabolic disease and benign prostate hyperplasia. Differentiation 82, 220-236 (2011).

11. Rosen, E. D. \& Spiegelman, B. M. PPARgamma: a nuclear regulator of metabolism, differentiation, and cell growth. J. Biol. Chem. 276, 37731-37734 (2001).

12. Werman, A. et al. Ligand-independent activation domain in the $\mathrm{N}$ terminus of peroxisome proliferator-activated receptor gamma (PPARgamma). Differential activity of PPARgamma1 and -2 isoforms and influence of insulin. J. Biol. Chem. 272, 20230-20235 (1997).

13. Jiang, M., Jerome, W. G. \& Hayward, S. W. Autophagy in nuclear receptor PPARgamma-deficient mouse prostatic carcinogenesis. Autophagy 6, 175-176 (2010).

14. Li T., et al. PPARG polymorphisms are associated with unexplained mild vision loss in patients with type 2 diabetes mellitus. J. Ophthalmol. 2019, 5284867 (2019).
15. Liu, C. et al. Pparg promotes differentiation and regulates mitochondrial gene expression in bladder epithelial cells. Nat. Commun. 10, 4589 (2019).

16. Merchan B. B., Tinahones F. J. \& Macias-Gonzalez M. Commonalities in the Association between PPARG and Vitamin D Related with Obesity and Carcinogenesis. PPAR Res. 2016, 2308249 (2016).

17. Yamada, Y. et al. A comprehensive analysis of allelic methylation status of $\mathrm{CpG}$ islands on human chromosome 21q. Genome Res. 14, 247-266 (2004).

18. Shen, L. et al. Genome-wide profiling of DNA methylation reveals a class of normally methylated CpG island promoters. PLoS Genet. 3, 2023-2036 (2007).

19. Feinberg, A. P. Phenotypic plasticity and the epigenetics of human disease Nature 447, 433-440 (2007).

20. Skvortsova, K, Stirzaker, C. \& Taberlay, P. The DNA methylation landscape in cancer. Essays Biochem. 63, 797-811 (2019).

21. Jiang, M. et al. Functional remodeling of benign human prostatic tissues in vivo by spontaneously immortalized progenitor and intermediate cells. Stem Cells 28, 344-356 (2010).

22. Bhatia, B. et al. Critical and distinct roles of p16 and telomerase in regulating the proliferative life span of normal human prostate epithelial progenitor cells. J. Biol. Chem. 283, 27957-27972 (2008).

23. Love, M. I., Huber, W. \& Anders, S. Moderated estimation of fold change and dispersion for RNA-seq data with DESeq2. Genome Biol. 15, 550 (2014).

24. Huang da, W., Sherman, B. T. \& Lempicki, R. A. Systematic and integrative analysis of large gene lists using DAVID bioinformatics resources. Nat. Protoc. $\mathbf{4}$ 44-57 (2009).

25. Livak, K. J. \& Schmittgen, T. D. Analysis of relative gene expression data using real-time quantitative PCR and the 2(-Delta Delta C(T)) Method. Methods 25, 402-408 (2001).

26. Subramanian, A. et al. Gene set enrichment analysis: a knowledge-based approach for interpreting genome-wide expression profiles. Proc. Natl Acad. Sci. USA 102, 15545-15550 (2005).

27. Brunetti, L., Loiodice, F., Piemontese, L., Tortorella, P. \& Laghezza, A. New approaches to cancer therapy: combining fatty acid amide hydrolase (FAAH) inhibition with peroxisome proliferator-activated receptors (PPARs) activation. J. Med. Chem. 62, 10995-11003 (2019).

28. Youssef, J. \& Badr, M. Peroxisome proliferator-activated receptors and cancer: challenges and opportunities. Br. J. Pharmacol. 164, 68-82 (2011).

29. Ngala, R. A. et al. A new, highly selective murine peroxisome proliferatoractivated receptor delta agonist increases responsiveness to thermogenic stimuli and glucose uptake in skeletal muscle in obese mice. Diabetes Obes. Metab. 13, 455-464 (2011)

30. Korbecki, J., Bobinski, R. \& Dutka, M. Self-regulation of the inflammatory response by peroxisome proliferator-activated receptors. Inflamm. Res. $\mathbf{6 8}$ 443-458 (2019).

31. Cesario, R. M., Stone, J., Yen, W. C., Bissonnette, R. P. \& Lamph, W. W. Differentiation and growth inhibition mediated via the RXR:PPARgamma heterodimer in colon cancer. Cancer Lett. 240, 225-233 (2006).

32. $\mathrm{Yu}$, J. et al. Inhibitory role of peroxisome proliferator-activated receptor gamma in hepatocarcinogenesis in mice and in vitro. Hepatology 51, 2008-2019 (2010).

33. Ahmad, I. et al. Sleeping Beauty screen reveals Pparg activation in metastatic prostate cancer. Proc. Natl Acad. Sci. USA 113, 8290-8295 (2016).

34. Rogenhofer, S. et al. Enhanced expression of peroxisome proliferate-activated receptor gamma (PPAR-gamma) in advanced prostate cancer. Anticancer Res. 32, 3479-3483 (2012)

35. Mirza, A. Z., Althagafi, I. I. \& Shamshad, H. Role of PPAR receptor in different diseases and their ligands: Physiological importance and clinical implications. Eur. J. Med. Chem. 166, 502-513 (2019).

36. Han, S. et al. MicroRNA-1251-5p promotes tumor growth and metastasis of hepatocellular carcinoma by targeting AKAP12. Biomed. Pharmacother. 122, 109754 (2020)

37. He, P. et al. Upregulation of AKAP12 with HDAC3 depletion suppresses the progression and migration of colorectal cancer. Int. J. Oncol. 52, 1305-1316 (2018).

38. Soh, R. Y. Z., Lim, J. P., Samy, R. P., Chua, P. J. \& Bay, B. H. A-kinase anchor protein 12 (AKAP12) inhibits cell migration in breast cancer. Exp. Mol. Pathol. 105, 364-370 (2018)

39. Aluru, N. et al. Developmental exposure to 2,3,7,8-tetrachlorodibenzo-p-dioxin alters DNA methyltransferase (dnmt) expression in zebrafish (Danio rerio). Toxicol. Appl. Pharmacol. 284, 142-151 (2015). 
40. Ceccarelli, V. et al. Molecular mechanisms underlying eicosapentaenoic acid inhibition of HDAC1 and DNMT expression and activity in carcinoma cells. Biochim. Biophys. Acta Gene. Regul. Mech. 1863, 194481 (2020).

41. Pu, M. et al. Regulatory network of miRNA on its target: coordination between transcriptional and post-transcriptional regulation of gene expression. Cell. Mol. Life. Sci. 76, 441-451 (2019).

42. He, M. et al. Down-regulation of miR-200b-3p by low p73 contributes to the androgen-independence of prostate cancer cells. Prostate 73, 1048-1056 (2013).
43. Moh-Moh-Aung, A. et al. Decreased miR-200b-3p in cancer cells leads to angiogenesis in HCC by enhancing endothelial ERG expression. Sci. Rep. 10, 10418 (2020).

44. Wu, J., Cui, H., Zhu, Z. \& Wang, L. MicroRNA-200b-3p suppresses epithelial-mesenchymal transition and inhibits tumor growth of glioma through down-regulation of ERK5. Biochem. Biophys. Res. Commun. 478 1158-1164 (2016). 